\title{
Adaptation of the Pedagogy in China Towards Innovation in Microelectronics
}

\author{
Olivier Bonnaud ${ }^{1,2,3}$ Lei Wei $^{3}$ \\ ${ }^{1}$ IETR, Institut d'Electronique et Télecommunications de Rennes, Université de Rennes 1, Rennes, France \\ ${ }^{2}$ GIP-CNFM, Groupement d'Intérêt Public-Coordination National pour la Formation en Microélectronique et Nanotechnologies, Grenoble, \\ France \\ ${ }^{3}$ Electrical Engineering Department, South-East University, Nanjing, China
}

Email address:

Olivier.bonnaud@univ-rennes.fr (O. Bonnaud)

\section{To cite this article:}

Olivier Bonnaud, Lei Wei. Adaptation of the Pedagogy in China Towards Innovation in Microelectronics. Science Journal of Education. Vol. 4, No. 2, 2016, pp. 65-72. doi: 10.11648/j.sjedu.20160402.18

Received: February 28, 2016; Accepted: March 8, 2016; Published: April 16, 2016

\begin{abstract}
The economical world needs constant innovation in order to develop new products. The field of microelectronics is particularly concerned with its very fast evolution in order to answer to the development of connecting objects that combine the strong improvement of the microelectronics technology and the applications to many domains. At the same time, the pedagogy in higher education moves progressively towards a numerical approach by involving more and more tools based on the Massive Open Online Courses (MOOC), internet sites, and simulation. The main challenge, today is to give to the students and future engineers, the methodology and the know-how that are not provided by the numerical tools in line, but also an innovative approach, with an improvement of the behavior of the educative structures and of the professorial body. The previous experience in China shows that basic education should change, on the one hand, in the learning of basic knowledge since the primary school by highlighting the understanding instead of the learning by heart, and on the other hand, by introducing practice and laboratory works, a way to develop the curiosity, the observation, the deep analysis, the links between many disciplines, the combination of several knowledge and the synthesis approach. However, the practice on technical platforms is very expensive and the sharing of this equipment between several institutions is necessary. The example of the French national network, CNFM (National Coordination for Education in Microelectronics and nanotechnologies) that pilots 12 national platforms, shows how to develop a high level practice with common platforms, and a policy deliberately focused on innovative practice on dedicated platforms. Several suggestions are given in order to improve the present China educational system and create a model which could be duplicated in many other countries.
\end{abstract}

Keywords: Higher Education, Innovation, Pedagogical Approach, Microelectronics, Practice in Science, Multidisciplinary Approach

\section{Context for the Innovation}

The innovation appears more and more mandatory in order to insure the survival of the companies in the world. It is clear that innovation is a way to create new products and to answer to the societal needs in many fields of applications [1] Many countries, like China, continuously manifest the need of innovation in their strategy. Following an expertise report, a special program was launched in 2006 by China's government in order to improve the innovation behavior of the new Chinese graduate students [2-3]. The innovation is expected to answer to many societal challenges and concerns many societal domains such as health, transport, energy, environment, communications, security [4], as shown figure 1 .

A part of this strategy is included in the program «1000 Talents» of Foreign Experts launched by the Chinese governement [5]. As one of the sub-programs of the "Recruitment Program of Global Experts" (1000 Talents Plan), «the Recruitment Program of Foreign Experts (RPFE) is to recruit non-ethnic Chinese experts, who are strategic scientists, leading experts in science and technology, or internationalized innovative teams capable of achieving critical technological 
breakthroughs, advancing the high-tech industries and promoting new disciplines" [citation from 5].

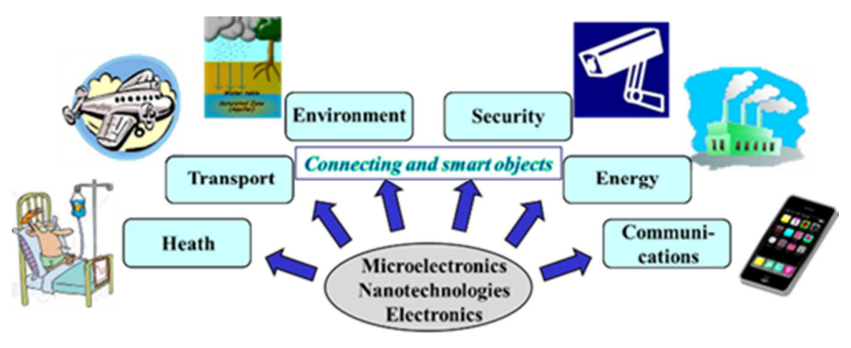

Figure 1. Evolution of microelectronics and nanotechnologies towards connecting objects. Many fields of application are concerned [4].

The new trends are to develop a lot of complex systems able to solve several global problems and find sustainable solutions for the future. We know that a lot of new concepts are applied, and tend towards a multiplication of the connecting objects as mentioned in the European strategy report [6] that plans the huge development of smart objects combined with the connecting possibilities. In the context of the tremendous evolution of the microelectronics technologies thanks to the reduction of dimensions [7], and more recently thanks to the hybridization of the technologies [8] towards heterogeneous systems [9], they are expected to give some better comfort, higher security of people and equipment, allowing control of the energy consumption, of the quality of the environment. They have also the goal to save life and to better adapt the technologies to the increase of the population and to the associated improvement of their direct environment [4]. For example, the new systems can protect the population against landslides and earthquakes [10] thanks to connecting properties. In the frame of the microelectronics, more and more objects are connected and smart, which means a combination of the intrinsic properties of electronics with application fields that can be quite diversified. Of course, the education must be adapted to this evolution in order to prepare the future engineers to their professional careers. More especially, a multidisciplinary awareness is increasingly useful for developing new objects [11]. This strategy is applied in the frame of the French national network for higher education in microelectronics and nanotechnologies with the goal to adapt the students to the new technologies [12-13] and prepare them to an innovative behavior [14].

Thus, this paper deals with innovation and education. After an analysis of the conditions to get an innovative behavior, on the base of the experience of the authors in China and in France in the education of microelectronics and of the new connecting objects, several suggestions are given, which are expected to better adapt the future doctors, engineers and graduate students of this field to this new technological world.

\section{How to Reach Innovative Behavior}

The innovation is not imposed by a law and its application decree. The innovation is generated by the environment, the societal culture, the cultural patrimony, and mainly by the educative approach. The first items are mainly due to the history, the evolution of the civilization. The last is much more recent, and is supposed to change with the societal evolution. However, the educative system involves a large amount of people who are educators, monitors, teachers, or professors from the primary school to the higher education, and a change of this whole category of population is not so easy due to a large inertia of the administrative structure, of the evolution of the careers, and of the direct contact with the population of the country.

Indeed, the innovation is first the result of the expression of the need by the economic environment, mainly companies that must encourage this skill. The companies have to recognize the skill not only by the salary but also by the work conditions in a research and development team, exchanges with other researchers in the world via conferences, seminars, but also exhibitions during which the societal needs are often well expressed [see for example, 1]. In parallel, the educators have to adapt the knowledge of the pupils, the schoolers, and the students, to the needs of the economic environment and to the evolution of behavior of the educated young in the society.

\section{Dependence on the Culture}

\subsection{Basic Education Focused on Memorization}

The innovative behavior is strongly dependent of the local culture and of the educational system. For the own experience of the first author, and from his knowledge of Chinese educational system, the pedagogical approach from primary school to the higher education, at least at the level of bachelor, is mainly based on the memorizing capabilities of the schoolers and students. Of course, learning Chinese language requires the students to make a strong effort to memorize the ideograms and their pronunciation. This is a mandatory pathway and the evaluation is based first of all on memorization capability.

\subsection{Evaluation Focused on Testing the Memorization Capability}

Unfortunately, the present Chinese system is focused on this point and whatever their teaching levels, and apparently whatever their discipline, the teachers are applying the principle that an exam is first of all built on capability to prove a level of knowledge. The exam subject is targeting mainly to prove that the young is able to learn by heart something and that he is able to repeat it exactly. Of course, this presentation is somewhere a caricature, but it is not so far from the reality. This means also that during their schooling, young are not selected, in average, on their capability of analysis, of understanding, and of synthesis.

\subsection{Limitations Due to the Memorization-Based Culture}

The memory-based education appears well adapted for production and reproduction of existing knowledge and know-how. However, this behavior is not sufficient to go 
ahead, more especially in the competitive world-wide economy. The challenge is to get an opening of the mind by confronting the memorized knowledge, by making links between the memorized data, by combining and confronting these data, by developing a critical mind, by solving new problems, and by a constructive analysis. In this way, innovative behavior becomes possible at least in the frame of scientific fields (the specialty of the authors).

Thus, the pedagogical approach must change in China even at the level of secondary school. The learning process must be partially devoted to the reflection on a real problem, to have effective practice with experiments on real equipment adapted to the new technologies, to suggest several solutions, and not to learn by heart a solution.

The question is now: what does China has to do in order to orient the future education within this objective?

\section{Apparent Limitations in the Chinese Educative System}

In addition of the cultural influence on the behavior of the young, the proposed approach, in fact, is not so easy, taking into account of the structural limitations, in other words of the educative system. There are apparently at least five main limitations.

\subsection{Evaluation of the Academic Institutions}

The first limitation seems to be the result of the present evaluation of Universities, for which the evaluation metrics, does not take into account the pedagogical activities of assistant-professors and professors. Indeed, in the evolution of the careers, today, more than ninety percent of the appreciation is based on the research activity and on the publication of results in international journals. The adaptation of the pedagogy to the students and to the evolution of technologies, the evolution of the contents of the lectures and the associated practice are not really considered as an important investment of the teachers, with a high added-value. One can notice, however, that, in the higher education, it is clear that the teachers must insure their life-long learning thanks to research activity. This corresponds to a permanent adaptation and to the renewing of the knowledge and the techniques. But, the mission of higher education professors is also to teach, and a part of the evaluation of the academic institutions, and of the professors and assistant professors, should take into account this activity, even if reliable indicators are not so easy to define. Several examples in European countries can be analyzed to set-up an equivalent approach in China. The Shanghai ranking of international Universities does not take into account this point. There seems to be a mistake in the metrics that should be changed.

\subsection{Basic Remuneration of the Teachers}

The second limitation is linked to the remuneration of the teachers, assistant-professors, and professors, in the higher education system that is a more global problem. The amount of base salary is not high enough to insure good standard of living, more especially in the large cities, while the teachers, with a very high level of instruction, should be in conditions that give them the possibilities to be fully devoted to their main missions of education and research. In fact, the teachers have to find complementary financial resources by supervising tens of internships (at master level) at the same time, in majority linked to companies. In the frame of conventions, the teachers can obtain financial supports, a part of which being directly destined for the supervisor. If this is a good way to get financial supports at the level of the institutions, it is not really a suitable strategy to improve the quality of life of the teachers, and above all, the quality of the education. In fact, the experience we have in the frame of electrical engineering in China, shows that the internship subjects are not chosen to prepare the students to research activities and to innovation, but in ninety percent of the cases, to make them solve the particular production problem of a company. Even if in the frame of the national economy, this type of subjects must exist, the objective of acquisition of know-how and skills adapted to innovative behavior will never be reached through this approach.

\subsection{Weakness of Practice in Foreign Language}

The third point corresponds to the organization of the studies with a very low amount of practice, of laboratory works (or labworks), and of exchanges with the professorial body. Indeed, many international experts [15] have revealed a lack of practice in the language learning, more especially a weak level of oral English among the Chinese students. This weakness is also visible in the frame of international diploma. The experience of the first author as expert of selection of students for the "masters 211" program [16] during several years and on the $1+1$ international master diploma that the authors have created between the South-East University and the "Université de Rennes 1" [17-18], is that even if the students seem to have a very good scientific knowledge, they are often not able to attend to the lectures given in English. In this case, they always learn by heart, and thus all the needed approach involving links and analysis, as previously mentioned, becomes inefficient [19].

\subsection{Weakness of the Linking the Knowledge in Science}

The fourth limitation is the result of weakness in the habit to link the knowledge between lectures of several disciplines and to link the theoretical learning with the practice. A recent personal experience, by setting up a module of specialty in microelectronics at the South-East University, entitled 'Integrated microelectronics technologies", at the level of the Bachelor, showed crucially this type of weakness in the acquired scientific knowledge of the students. During the first lectures, given with another methodology that consists to permanently raise questions, and to make links with a lot of disciplines, many students are surprised and even partially lost. 
In fact, the students have learnt by heart many contents of lectures but they have not made in general the links between many disciplines that are in fact strongly linked in the real objects. Because they are usually bright, when they have really understood the role and the interest of the new approach, they start to appreciate this change in pedagogical approach [19-20]. The best students acquire the capability to prepare a $\mathrm{PhD}$ abroad, in very good conditions. The first author has this experience with a student of South-East University who has prepared a thesis under his supervision in France, in the Department of Microelectronics and Sensors of his laboratory, IETR. The student proved to be one of the best students that he had never supervised before.

\subsection{Weakness of Practice in Science}

The practice and laboratory works (or labworks) in science are the best way to explain deeply the reality of things and industrial objects. The main philosophy should consist to highlight the fundamental mechanisms or properties, while nowadays and with an almost equivalent need, it is necessary to estimate the limitations, the domain of validity of the modelling and simulations, and the mission profile that also affects the quality and the reliability of the final product. This is becoming a key factor to make comparison between the theoretical approach and the real features and, above all, a way to find new ideas to better control all these parameters.

Figure 2 summarizes the emphasized limitations of the Chinese educational organization that are presented above. The left hand part is devoted to the influence of the culture. The right hand part is devoted to the effect of the education system. Several of these limitations can be overpassed easily. However, several remain very difficult to change in a short time.

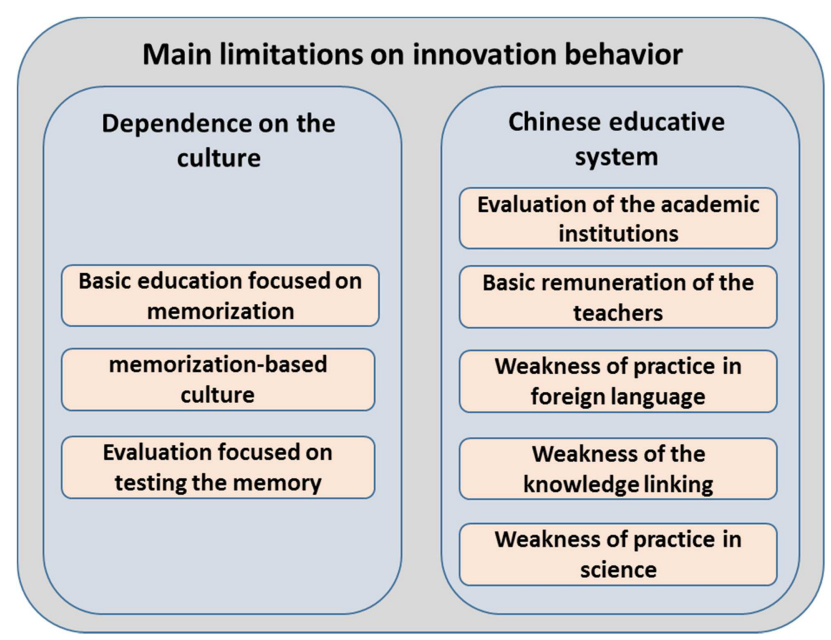

Figure 2. Main limitations to the innovating behavior of the Chinese students. Several of these limitations can be overpassed easily.

\section{General Suggestions of Improvement}

Hereafter, several points that were highlighted above in order to induce an improvement are summarized. They could lead to an evolution of the skills of the students towards innovation.

\subsection{Modification of the Secondary School Pedagogy}

In order to accustom students to apply the theoretical knowledge, the pedagogy at the level of the secondary school should evolve by introducing evaluation on the understanding via problems. These problems must be designed in order to well train the schoolers, but in the same time to link the study to a real situation or to a physical object. This evolution appears as a huge work. The first step should consist to train the teachers of secondary schools and to convince them of the interest of this approach. The second step should consist to progressively modify the mode of selection of students, which can be oriented towards new types of exams, not only based on the learning by heart of a knowledge, mainly, but also on the resolution of problems, more especially to enter at the University through Gao Kao exam.

\subsection{Modification of the Higher Education Pedagogy}

This change of behavior appears less difficult to engage than the previous one at the levels of Bachelor and master because the professorial community in the Higher Education is smaller and the students can be more easily adapted. More mature, they are able to understand the importance of the modification of the behavior. However, this evolution depends of other factors that are also mentioned in this enumeration.

\subsection{Modification of the Academic Institution Evaluation}

The evaluation of the institutions appears today as a limitation of the evolution of the pedagogy. Indeed, the ranking of the academic institutions depends mainly on the research activities, on the research results, and on research recognition. It should be important to modify the present metric of evaluation of the Institutions by increasing the role of the pedagogical activity, while maintaining a balance between the teaching and the research activities.

\subsection{Better Remuneration of the Higher Education Teachers}

An increase of the remuneration by the organisms and Institutions of the professorial community should be engaged, but in return of the limitation of the number of extra lectures that consist in the majority of the cases to be a simple repetition, and of the number of internships simultaneously supervised. At all the levels, this activity of supervising could be shared, even with the young teachers, or assistant-professors. Without the financial pressure, the situation could be much better in the Institutions.

\subsection{Learning by Problems}

The students should have a training on how to solve problems at each level of the education. It is important to mention that in many real cases, the solution is not unique, and the approach either. This is a way to increase the diversity of thinking, which is a key for the innovative behavior. 


\subsection{Learning by Projects}

The increase of participation of the students in the learning process is a way to make them aware of the discipline and to be much more motivated to make an effort of understanding. Thus, more interactive classes should be organized and the learning by projects should also be developped by teachers. This is the counterpart of the decrease of lectures in amphitheaters and a way to be much more incentive for the reflection and the analysis capability of the students.

\subsection{Development of Practice}

As already mentioned, the practice, corresponding to labworks and to projects, is more and more important in the curriculum of the graduate students and more especially of the engineers. This practice should be enhanced whatever the domain of study. Depending of the domain, the associate investment can be significantly different. If in the frame of language learning, the equipment of specific laboratories is rather inexpensive, in the frame of scientific fields the investment can be huge. This should not constitute an obstacle if a collective strategy is built.

To summarize, the different points on which actions could be developed are shown on figure 3. On the left hand side, the actions on the educative system and on the right hand side, the actions of the pedagogical approach. One can notice that in the field of microelectronics, the creation of technological platforms, especially devoted for education and that can be common between several academic institutions is introduced in the figure. This point is developed in the next paragraph.

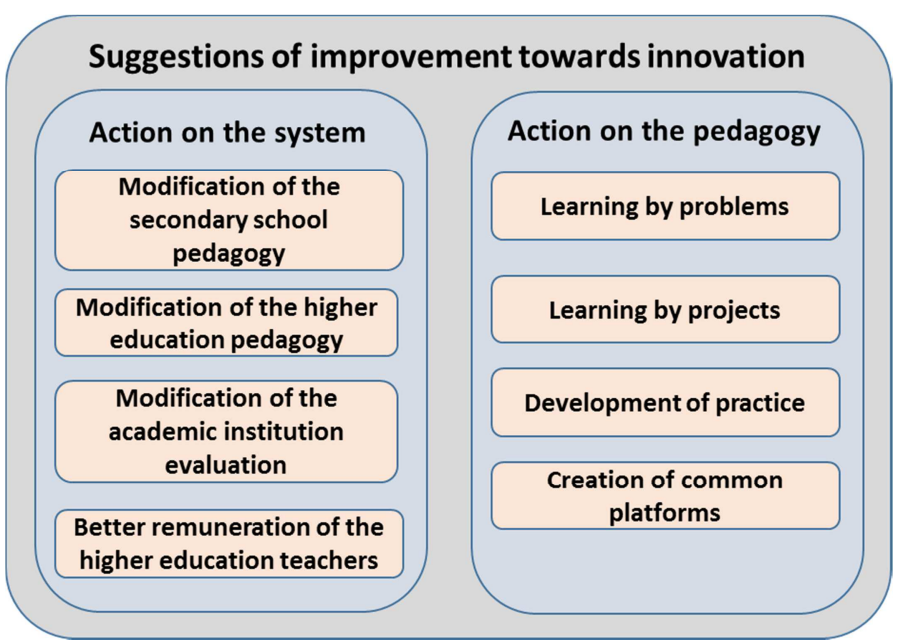

Figure 3. Suggestions that could be applied in order to improve the innovative behavior thanks to an evolution of the structure and of the pedagogical methodology.
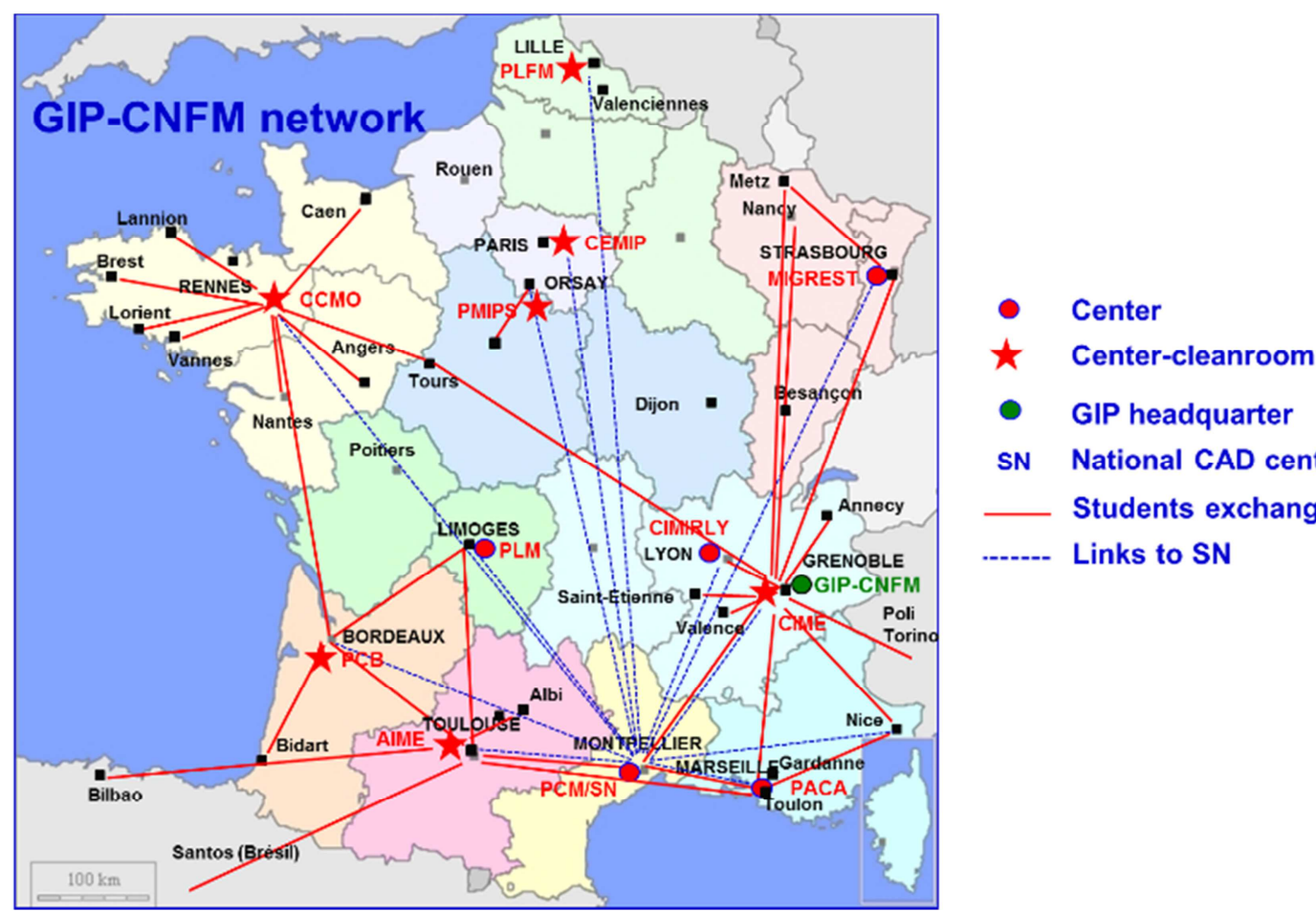

- GIP headquarter

SN National CAD center

Students exchanges

Links to $\mathrm{SN}$

Figure 4. French Microelectronics network. The 12 centers are common with several academic institutions [12]. 7 centers have cleanroom facilities. 


\section{Development of Innovation in Microelectronics}

\subsection{Strategy of Sharing the Investment: A National Network}

In order to insure the formation on up-to-date equipment, more especially in microelectronics, the only suitable solution consists to organize a network, as it was done in France since more than thirty years, with platforms common between several institutions $[12,21]$. The network is recognized by the Ministry of Higher Education, and is composed of 12 national centers that are common with several higher education institutions of the geographic zone. The administrative form is a Public Interest Group, entitled "GIP-CNFM" for "National Coordination for Education in Microelectronics and Nanotechnologies" [12]. Figure 4 shows the geographic implantation of the centers with their links, more especially for the computer-aided design activity.

The sharing of the investment and the functioning expenses are mandatory. Indeed, a cleanroom for the microelectronic technology education, needs an investment close to 40 million RMB. This amount is however several hundred time lower than a production line in a company! The tools for Computer-Aided-Design (CAD) of microelectronics or nanoelectronics circuits are very expensive and they need adapted servers with a high capacity of storage and treatment. Behind this, the maintenance needs more or less 5 million RMB per year of functioning expenses, for which one can add in average, the technical support of engineers and technicians that can be in the range of 5. The optimization of the investment and of the functioning expenses leads clearly to the sharing of these platforms between several higher education institutions at local level or at the level of a Province. The annual number of users (students), whatever their level, must reach 1.000 to 1.500 corresponding to 100.000 hours-students, in order to achieve an hourly cost low enough and compatible with the budget of educational departments of the universities.

\subsection{Strategy of Innovation in Microelectronics}

The very fast evolution of this domain that follows, more or less, the Moore's Law [7] for the integration, and "More than Moore's Law" [8-22] for the complexity of the systems, imposes a permanent adaptation of the practice. Thus, the strategy of the French network includes an incentive policy for innovation, through annual calls for new training projects in agreement with the surveys and expertise reports on the prospective [23]. Since 2008, the strategy of the GIP-CNFM has consisted to insert the innovation in the policy and to apply for complementary financial supports to several programs such as Nano-Innov [24] and IDEFI-FINMINA [25] deliberately oriented towards pedagogical innovation in the discipline. These innovative labworks were the results of the research activities of the professorial body and concerned the evolution of the integration in the scheme of "Moore's Law" but also the new integrations of systems with their applications to many domains in the scheme of "More than Moore's Law".

\subsection{Results of These Strategies for the French Network}

This strategy allowed the creation of many new practices as reported in several activities reports and in journal papers [26-27], as well as on the website of the CNFM network [12]. Each academic year, about 3.500 students have the opportunity to have practices on the platforms on these innovative labworks. After five years, more than sixty innovative practices were created in the national network.

\section{Opportunity of Changing the Education Approach}

The introduction in the education of numerical tools such as MOOCs [28-29] associated to a deep change of behavior of the young with the surrounding numerical world, are creating the conditions to deeply modify the general pedagogical approach. The number of lectures in large amphitheater will decrease to the benefit of learning in small groups by projects, by games, or by proactive classes. In this case, after a first learning by MOOCs devoted to the specialty, for example [29-30], a small group of students should work with a teacher with the goal to solve problems and to go deeper in the reflection and in the methodology.

\section{Conclusion}

In the field of microelectronics and nanotechnologies, a domain with a very fast evolution and needing a permanent innovative approach, China's education system has displayed several weaknesses and several limitations. These two problems seem to be coming from the cultural environment and from the pedagogical approach that does not highlight the analyses of problems, and above all the practice. Several suggestions are given that should improve the skills of the students towards an innovative behavior. These suggestions concern as well the organization of the educational system as the modification of the pedagogical methodology. A large part of the future activities of the students should provide an increase of labworks, projects, problem solving, and internships. This approach was developed for many years in the frame of the CNFM French network and has proved its efficiency. However, this implies a strong evolution of the educational system, with a new type of evaluation of the students as well as of their teachers. The present tendency combined to the emergence of pedagogical tools can give very interesting opportunities, likely to motivate the professorial community and to convince her to engage in this evolution. These suggestions can be applied in many other countries that want to orient the studies in microelectronics and nanotechnologies towards innovation. 


\section{Acknowledgements}

The authors want to thank all the members of "GIP-CNFM" network, the colleagues of South-East University and Université de Rennes 1 that are involved in the common international activities of research and teaching. This work is in part financially supported by French Higher Education Ministry I, by IDEFI-FINMINA program (ANR-11-IDFI-0017) and by the Chinese Government in the frame of "1000 Talents" Foreign Expert program. A special thanks to L. Chagoya-Garzon, secretary of GIP-CNFM for her fruitful advice for the redaction of this paper.

\section{References}

[1] G. Matheron, Keynote, Microelectronics evolution, European Microelectorncis Summit, Paris, Nov. 2014

[2] J Guan, S Liu, "Comparing regional innovative capacities of PR China based on data analysis of the national patents", International Journal of Technology, 2005, inderscienceonline.com

[3] JC Guan, RCM Yam, CK Mok, "Collaboration between industry and research institutes/universities on industrial innovation in Beijing, China", Technology Analysis \& Strategic Management, Volume 17, Issue 3, 2005

[4] O. Bonnaud, L. Fesquet, Multidisciplinary topics for the innovative education in microelectronics and its applications, Proc. of, ITHET'15 Lisboa (Portugal), DOI: 10.1109/ITHET.2015.7217961, pp.1-5, 2015

[5] "Recruitment Program of Global Experts" (1000 Talent Plan): http://1000plan.safea.gov.cn/

[6] Strategic research agenda of EPOSS - the European technology platform on smart systems integration, http://www.smart-systems-integration.org/public/documents/p ublications

[7] G.E. Moore, Cramming more components onto integrated circuits, Electronics Magazine, 38 (8), pp.114-117 (1965)

[8] "More-than-Moore" White Paper. Editors: W. Arden, M. Brillouet, P. Cogez, M. Graef, B. Huizing, R. Mahnkopf, International Roadmap Committee of ITRS, 2010, www.itrs. net/Links/2010ITRS/IRC-ITRSMtM-v2\%203.pdf

[9] M. Swaminathan, J. M. Pettit, 3rd System Integration Workshop, 2011, Available: http://www.ipc.gatech.edu/workshop/2011/madhavan.pdf

[10] Y. Lami, H. Mediouni, D. Genon-Catalot, A. Lagrèze, D. Jongmans, L. Baillet, D. Donsez, Landslides prevention using a buried wireless sensors network, ISNPEDAM'2015, La Réunion (France), Oct. 2015, accepted for publication in International Journal of Plasma Environmental Science \& Technology, 2016

[11] O. Bonnaud, New approach for sensors and connecting objects involving microelectronic multidisciplinarity for a wide spectrum of applications, ISNPEDAM'2015, La Réunion (France), Oct. 2015, accepted for publication in International Journal of Plasma Environmental Science \& Technology, 2016

[12] CNFM: Coordination Nationale pour la formation en
Microélectronique and nanotechnologies (National Coordination for Education in Microelectronics and Nanotechnologies). website: www.cnfm.fr; GIP-CNFM : Public Interest Group, administrative structure of the CNFM network

[13] O. Bonnaud, P. Gentil, A. Bsiesy, S. Retailleau, E. Dufour-Gergam, J. M. Dorkel, "GIP-CNFM: a French education network moving from microelectronics to nanotechnologies", Oral communication; Proc. of IEEE of EDUCON'11; Amman (Jordan) 3-6 April 2011, ISBN978-1-61284-641-5, pp. 122-127

[14] O. Bonnaud, L. Fesquet, P. Nouet, T. Mohammed-Brahim, "FINMINA: a French national project to promote Innovation in Higher Education in Microelectronics and Nanotechnologies", ITHET 2014 York, Proc. loaded at http://www.york.ac.uk/conferences/ithet2014/ paper 74 session 2

[15] "1000 Talents" foreign experts meeting, Beijing (China), 5 February 2016

[16] Master 211 - Chinese Universities - Project 211 is a project of National Key University and college initiated in 1995 by the Ministry of Education of the China, with the intent of raising the research standards of high-level universities and cultivating strategies for socio-economic development

[17] O. Bonnaud, Set-in an Asian Remote International Master in Electrical and Information Engineering on the Base of the Bologna Process, Communication oral; EAEEIE'07; Prague (Czech Rep.); ISBN 978-80-01-03-745-4, session T2B, 5 pages, 2-4 July 2007

[18] O. Bonnaud, L. Senhadji, H. Fremont, L. Wei, H. Shu, L. Limin, Field return on a Chinese-French double graduation of an International Master in Electronics and Telecommunications on the Base of the Bologna Process, Oral communication; ITHET'10; Urgup (Turkey) 29 April-1 May 2010, CD-ROM, Proc. ISBN: 978-1-4244-4811-1, pp.324-327, 2010

[19] O. Bonnaud, Difference of pedagogical approaches for Chinese and French master students in a French-Chinese microelectronics joint master diploma. Oral communication, Proc. ITHET'15 Lisboa (Portugal), 11-13 June, Proc. DOI: 10.1109/ITHET.2015.7217962, pp. 1-4, 2015

[20] O. Bonnaud and X. Zhong, Adaptation of the Pedagogical Approaches for master students in Microelectronics in the Frame of a French-Chinese Joint Program. Proc. of SBMicro'2015, Salvador de Bahia (Brazil), 1-4 Sep, DOI: 10.1109/SBMicro.2015. 7298143, 4 pages, 2015

[21] O. Bonnaud, G. Rey, "The French microelectronics training network supported by industry and education ministries", Proc. IEEE Int. Conf. on Microelectronic Systems Education (MSE'97), DOI: 10.1109/MSE.1997.612575, pp. 121-122, 1997

[22] M. Orlowski and A. Wild, Can 3-D devices extend Moore's Law. Beyond the $32 \mathrm{~nm}$ technology node" Electrochem. Soc Transactions, 3 (6), 1, (2006)

[23] STranES. French report: Stratégie nationale de l'enseignement supérieur, www.enseignementsup-recherche.gouv.fr/pid30540/ www.enseignementsup...

[24] NanoInnov: French Innovation program on Nanotechnologies, including a part for the development of Education, in the frame of the "Grand Investissement" pluriannual program of the French government 
[25] FINMINA: Formations Innovantes en Microélectronique et Nanotechnologies (Innovation Education in Microelectronics and Nanotechnologies). See website of CNFM, IDEFI project: ANR-11-IDFI-0017

[26] O. Bonnaud and L. Fesquet, Innovating projects as a pedagogical strategy for the French network for education in microelectronics and nanotechnologies, Proc. of IEEE Int. Conf. on Microelectronic Systems Education (MSE'13), Print ISBN: 978-1-4799-0139-5, pp. 5-8, 2013

[27] O. Bonnaud, L. Fesquet, Multidisciplinary topics for the innovative education in microelectronics and its applications,
Oral communication, ITHET 2015 Lisboa (Portugal), 11-13 June, 2015

[28] O. Bonnaud, Panel session: Future educational challenges in electrical engineering education: Will MOOCs be a threat or an opportunity? Round Table Panelist; EWME 2014, Tallinn (Estonia), 14th-16th May, 2014

[29] O. Bonnaud et al., Microelectronics Online course: https://microelectronique.univ-rennes1.fr/index21.html

[30] https://www.mooc-list.com/course/computer-aided-design-cad -saylororg?static=true, Computer-Aided Design (CAD) 